\title{
Relieving the Hubble Tension with Primordial Magnetic Fields
}

\author{
Karsten Jedamzik ${ }^{1, *}$ and Levon Pogosian $\oplus^{2,3, \dagger}$ \\ ${ }^{1}$ Laboratoire de Univers et Particules de Montpellier, UMR5299-CNRS, Universite de Montpellier, 34095 Montpellier, France \\ ${ }^{2}$ Department of Physics, Simon Fraser University, Burnaby, BC V5A 1S6, Canada \\ ${ }^{3}$ Institute of Cosmology and Gravitation, University of Portsmouth, Portsmouth PO1 3FX, United Kingdom
}

(Received 28 April 2020; revised 20 July 2020; accepted 10 September 2020; published 28 October 2020)

\begin{abstract}
The standard cosmological model determined from the accurate cosmic microwave background measurements made by the Planck satellite implies a value of the Hubble constant $H_{0}$ that is 4.2 standard deviations lower than the one determined from type Ia supernovae. The Planck best fit model also predicts higher values of the matter density fraction $\Omega_{m}$ and clustering amplitude $S_{8}$ compared to those obtained from the Dark Energy Survey Year 1 data. Here we show that accounting for the enhanced recombination rate due to additional small-scale inhomogeneities in the baryon density may solve both the $H_{0}$ and the $S_{8}-\Omega_{m}$ tensions. The additional baryon inhomogeneities can be induced by primordial magnetic fields present in the plasma prior to recombination. The required field strength to solve the Hubble tension is just what is needed to explain the existence of galactic, cluster, and extragalactic magnetic fields without relying on dynamo amplification. Our results show clear evidence for this effect and motivate further detailed studies of primordial magnetic fields, setting several well-defined targets for future observations.
\end{abstract}

DOI: 10.1103/PhysRevLett.125.181302

The standard $\Lambda$ cold dark matter $(\Lambda \mathrm{CDM})$ model of cosmology has withstood two decades of testing against the ever improving observational data. However, with multiple independent types of measurements producing very accurate results over the past few years, some tensions between the $\Lambda \mathrm{CDM}$ parameters obtained from different datasets have emerged. Most notable of them is the discrepancy between the value of the Hubble constant $H_{0}$ inferred from the cosmic microwave background (CMB) measurements by Planck [1] and the one obtained from type Ia supernovae (SNIa) and certain other types of measurements in the $z \sim 0.01-1$ redshift range. In particular, the Planck best fit value of $H_{0}=$ $67.36 \pm 0.54 \mathrm{~km} \mathrm{~s}^{-1} \mathrm{Mpc}^{-1}$ [1] agrees very well with $H_{0}=67.4_{-1.2}^{+1.1} \mathrm{~km} \mathrm{~s}^{-1} \mathrm{Mpc}^{-1}$ obtained from the Dark Energy Survey Year 1 (DES-Y1) clustering and weak lensing data combined with baryon acoustic oscillation (BAO) measurements from a variety of spectroscopic surveys [2]. But it is significantly $(4.2 \sigma)$ lower than $H_{0}=73.5 \pm 1.4 \mathrm{~km} \mathrm{~s}^{-1} \mathrm{Mpc}^{-1}$ measured by the Supernovae H0 for the Equation of State of Dark Energy (SH0ES) Collaboration [3] using SNIa luminosities calibrated on Cepheid variable stars. SNIa studies using alternative calibration methods also find higher values of $H_{0}$ [4-7] (see Ref. [8] for a discussion). Determinations of $H_{0}$ that do not rely on SNIa include the Megamaser Cosmology Project (MCP) [9] that obtained $H_{0}=73.9 \pm 3.0 \mathrm{~km} \mathrm{~s}^{-1} \mathrm{Mpc}^{-1}$ [10] from very-long-baseline interferometry observations of water masers orbiting supermassive black holes, and the HO Lenses in COSMOGRAILs Wellspring (HOLiCOW) value of $H_{0}=73.3_{-1.8}^{+1.7} \mathrm{~km} \mathrm{~s}^{-1} \mathrm{Mpc}^{-1}$ [11] inferred from a joint analysis of six gravitationally lensed quasars with measured time delays.

Another, albeit somewhat weaker, tension exists between the values of the amplitude of galaxy clustering $S_{8}$ and the matter fraction $\Omega_{m}$ in the Planck best-fit model and those inferred from the latest surveys of large scale structure. Specifically, the Planck values are $S_{8}=0.832 \pm 0.013$ and $\Omega_{m}=0.3153 \pm 0.0073$ [1], while the DES-Y1 weak lensing and galaxy clustering data yields $S_{8}=0.783_{-0.025}^{+0.021}$ and $\Omega_{m}=0.264_{-0.019}^{+0.032}[12]$.

Many extensions of the $\Lambda \mathrm{CDM}$ model have been proposed with the aim of resolving the $H_{0}$ problem (see Ref. [13] for a review). Late-time dynamical dark energy or modifications of gravity can reduce the tension [14-17], but there is no evidence for them otherwise and, aside from the higher value of $H_{0}$, the dynamics of the Universe in the $z \sim 0.1-1$ redshift range is in good agreement with $\Lambda \mathrm{CDM}$. Importantly, a higher $H_{0}$ is preferred by all measurements that do not rely on our understanding of the recombination history and the determination of the sound horizon at the photon and the baryon decoupling epochs. If the sound horizon at recombination happened to be smaller due to a yet unaccounted effect, the observed angular acoustic scales in the CMB anisotropies and galaxy density fluctuations would imply a larger value of $H_{0}$. This would happen, for example, if the dark energy density became dynamically important for a period of time before recombination [18-21]. However, as recently pointed out in Ref. [22], such early dark energy (EDE) would delay the development of gravitational potentials, requiring a 
larger matter density to compensate and worsening the $S_{8}-\Omega_{m}$ tension. The situation may be better when considering modifications in the neutrino sector of the standard model [23].

In this Letter we show that both the $H_{0}$ and the $S_{8}-\Omega_{m}$ tensions may be greatly alleviated when allowing for smallscale, mildly nonlinear inhomogeneities in the baryon density shortly before recombination. Such baryon inhomogeneities are motivated by detailed studies of the evolution of primordial magnetic fields (PMFs) before recombination [24,25]. In a nutshell, on scales well below the photon mean free path (and the Silk damping scale) the effective speed of sound is far lower than that of a relativistic plasma such that PMFs of relatively moderate $\sim 0.1$ nano-Gauss (nG) strength [26] can generate baryon inhomogeneities on $\sim \mathrm{kpc}$ scales. Based on a comprehensive understanding of the PMF evolution in the early Universe [27], the effect has been derived analytically [24] and confirmed numerically [25]. In fact, the latter study used the Planck 2013 CMB data with a variety of other datasets to stringently constrain PMFs.

Even though small-scale baryon inhomogeneities on $\sim \mathrm{kpc}$ scales do not directly source CMB temperature and polarization anisotropies, their existence impacts the observed $\mathrm{CMB}$ anisotropies by profoundly changing the ionization history, and therefore the epoch of recombination. The ionization fraction $\chi_{e}$ is determined by a balance between the recombination rate, proportional to the electron density square $n_{e}^{2}$, and the ionization rate proportional to the neutral hydrogen density $n_{H}$. As any inhomogeneous universe has $\left\langle n_{e}^{2}\right\rangle>\left\langle n_{e}\right\rangle^{2}=\left.\left\langle n_{e}^{2}\right\rangle\right|_{\text {homo }}$, the average recombination rate is increased and the recombination occurs earlier in an inhomogeneous universe. An earlier recombination, in turn, reduces the sound horizon $r_{*}$ at recombination. The corresponding impact on the CMB anisotropy spectra would be a shift of the locations of all the acoustic peaks to smaller scales. Since the positions of the peaks, $\ell_{p} \propto r_{\mathrm{ls}} / r_{*}$, are measured with great accuracy by Planck, one would need a smaller conformal distance to last scattering $r_{\mathrm{ls}}$ to compensate for the shift, which requires a larger $H_{0}$.

In Refs. [24,25] a baryon clumping factor $b=\left(\left\langle n_{b}^{2}\right\rangle-\right.$ $\left.\left\langle n_{b}\right\rangle^{2}\right) /\left\langle n_{b}\right\rangle^{2}$ was introduced to gauge the amplitude of the PMF generated inhomogeneities. Since the recombination term is quadratic, one could naively expect that the reduction of the ionization fraction in inhomogeneous universes is fully determined by the average density and the clumping factor alone, i.e., the first two moments of the baryon density probability distribution function (PDF). However, a more careful analysis shows that the reduction of $\chi_{e}$ depends on all moments of the baryon density PDF. In the absence of detailed knowledge of the PDF, we employed two different PDFs implemented via a threezone model described below.

We have modified the publicly available code for anisotropies in the microwave background (CAMB) [28] to include the effects of small-scale baryon inhomogeneities on the recombination history. In particular, we make the code RECFAST [29-31] calculate the evolution of $\chi_{e}$ independently in three different zones, with the electron density in each zone drawn from a PDF normalized to set values of $\left\langle n_{b}\right\rangle$ and $b$, and take the appropriate average. The independent-zone approximation is well justified, since the $\sim 1 \mathrm{kpc}$ length scale corresponding to the clumping effect is much bigger than the mixing scale of $\sim 1 \mathrm{pc}$ (comoving) set by the diffusion length of baryons at recombination. Turbulent mixing due to MHD turbulence, on the other hand, is absent, since the plasma is in a low Reynolds number viscous state due to the strong residual photonelectron interactions.

Having three zones keeps the computational costs down, while still demonstrating the importance of accounting for the shape of the baryon density PDF. We have chosen two distinct distributions, hereafter referred to as model 1 (M1) and model 2 (M2), detailed in the Supplemental Material [32]. M1 is the model used in Refs. [24,25], while M2 was designed to show that the impact on recombination can be weaker despite the PDF having the same second moment $b$. In M2, only a tiny fraction of the total volume is in high density regions, with more of the remaining volume at densities close to the average. Models M1 and M2 approximately bracket the possibilities of a large number of three-zone models with the same average baryon density and clumping factor that we tried.

Having modified CAMB, we use CosmoMC [33] to generate Markov chains and find the marginalized posterior distributions for the cosmological parameters in the presence of baryon clumping. We use the Planck 2018 temperature, polarization, and CMB lensing spectra (TT,TE,EE+lowE+ lensing of Ref. [1]) hereafter called "Planck," and the three determinations of $H_{0}$ by SHOES, MCP, and HOLiCOW referred to as " $H 3$."

Figure 1 shows the marginalized posterior distributions for $b$ and $H_{0}$. Whereas we find no preferred clumping when using only the CMB data, as in Ref. [25] (see the Supplemental Material [32]), one can see that after including the three $H_{0}$ determinations into the analysis the marginalized posterior probability clearly prefers clumping of the order $\sim 0.5$. Moreover, as expected, due to the decreased sound horizon, the preferred value of the Hubble constant is larger and in better agreement with the $H_{0}$ observations. A further increase in clumping seems to be ruled out by the CMB data as it probably results in unacceptably large distortions of the Silk damping tail, precluding higher values of the inferred $H_{0}$. While zero clumping is essentially ruled out for both M1 and M2, Fig. 1 demonstrates the fairly large dependence of the effect on the yet unknown baryon density PDF. A more detailed investigation is under way.

Further results of the effects of clumping on current cosmological tensions may be observed in Fig. 2. The M1 

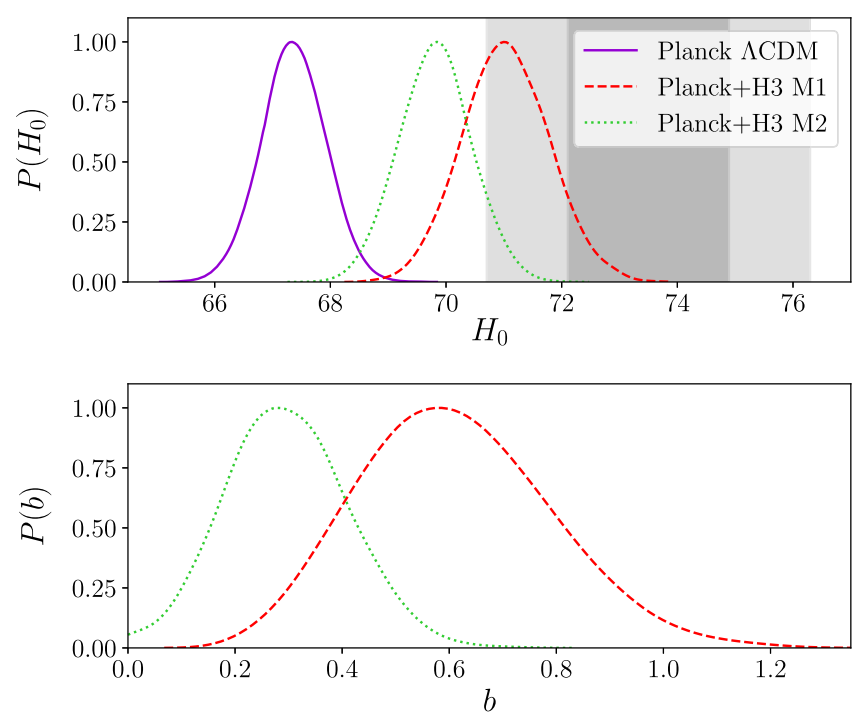

FIG. 1. The marginalized $H_{0}$ PDF for the Planck best fit $\Lambda \mathrm{CDM}$ model and the two baryon clumping models, M1 and M2, fit to Planck combined with H3. The bottom panel shows the PDF of the clumping parameter $b$. The shaded regions show the $68 \%$ and $95 \%$ C.L. of $H_{0}$ from SHOES [3].

(M2) clumping model prefer $\Omega_{m}=0.2873 \pm 0.0064$ $(0.2926 \pm 0.0064) \quad$ and $\quad S_{8}=0.809 \pm 0.012 \quad(0.809 \pm$ 0.012), significantly lower than in the Planck best fit $\Lambda \mathrm{CDM}$ model and in good agreement with the values determined from the DES-Y1 weak lensing and galaxy clustering data independent of recombination physics. The possible resolution of both tensions by one well-motivated physical addition to $\Lambda \mathrm{CDM}$, small-scale baryon clumping, is graphically presented in the right panel of Fig. 2. Depending on the baryon PDF, the Hubble constant tension is reduced from $4.2 \sigma$ to $\lesssim 2 \sigma(\lesssim 3 \sigma)$ for M1 (M2), whereas the $S_{8}-\Omega_{m}$ tension is removed.

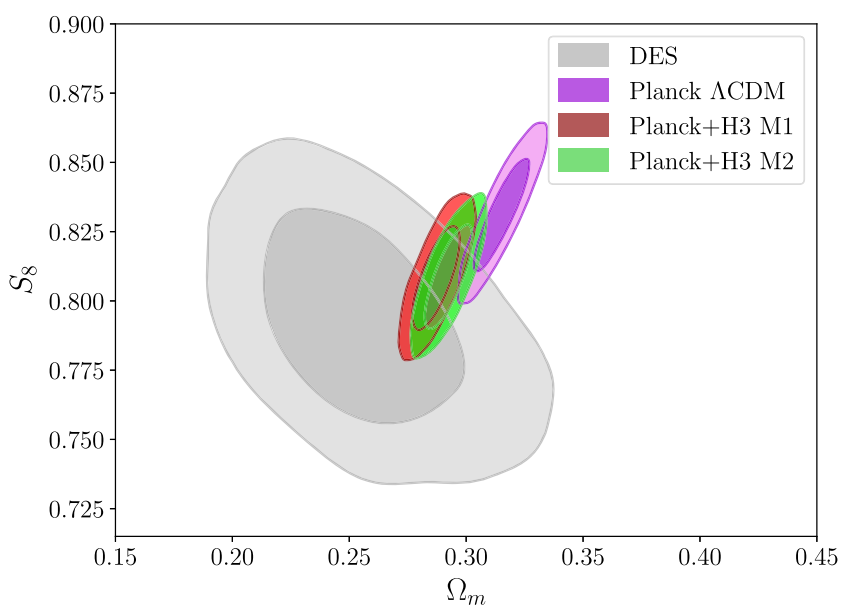

How does the addition of baryon clumping affect the goodness of fit to the Planck CMB data? One can see in Fig. 1 of the Supplemental Material [32] that without allowing for clumping, $\Lambda \mathrm{CDM}$ prefers a lower value of $H_{0}$ even after adding the $H 3$ data. The statistical weight of the $\mathrm{CMB}$ dataset, which is both very large and very precise, is much higher than that of the three $H 3$ points and adding the latter has limited impact on cosmological parameters. Allowing for clumping using model 1 makes the decisive difference, moving the best fit to $H_{0}=71.03 \pm$ $0.74 \mathrm{~km} \mathrm{~s}^{-1} \mathrm{Mpc}^{-1}$ and a nonzero clumping value of $b=$ $0.61_{-0.20}^{+0.16}$ at $68 \%$ C.L. $\left({ }_{-0.33}^{+0.35}\right.$ at $95 \%$ C.L. $)$, while model 2 gives $H_{0}=69.81 \pm 0.62 \mathrm{~km} \mathrm{~s}^{-1} \mathrm{Mpc}^{-1}$ and $b=0.31 \pm$ 0.11 at $68 \%$ C.L. $( \pm 0.22$ at $95 \%$ C.L. $)-\mathrm{a} 4 \sigma(3 \sigma)$ detection of clumping in M1 (M2). The mean $\chi^{2}$ of the $l>29$ TT, TE, and EE binned multipoles portion (the "Plik" part) for the Planck $+\mathrm{H} 3$ best-fit M1 model is larger than that of the Planck $\Lambda$ CDM by 6.7, which is comparable to the $1 \sigma$ uncertainty in $\chi_{\text {plik }}^{2}$ (see Table I in the Supplemental Material [32]). This means that Planck $+\mathrm{H} 3 \mathrm{M} 1$ is essentially as good a fit to CMB as the Planck $\Lambda$ CDM. A good statistical measure for judging the goodness of fit of a particular model to the CMB data is the probability to exceed (PTE). Assuming the model is correct, the PTE quantifies the probability of statistical fluctuations in the data resulting in a worse fit. Taking only the Plik likelihood, the Planck Collaboration finds PTE $\approx 0.2$ for their best-fit model (Table 20 of Ref. [34]). This drops to a PTE of $\approx 0.17-0.16$ for both the Planck $+\mathrm{H} 3$ best-fit M1 and M2, hardly a serious degradation. However, it is the latter models that alleviate the two existing tensions. The change in $\chi^{2}$ for the Planck lower non-Gaussian multipoles and CMB lensing is minor.

The impact of including additional datasets [12,35-39] is discussed in the Supplemental Material [32]. In particular, adding the BAO data [40]tend to reduce the value of $H_{0}$ to

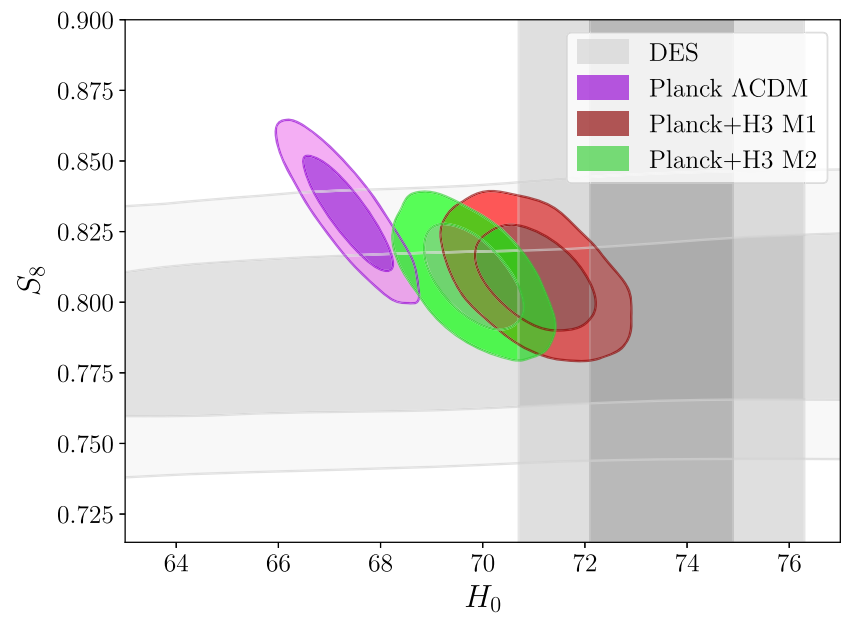

FIG. 2. The marginalized PDF contours for $S_{8}-\Omega_{m}$ (left) showing the mild tension between Planck and DES Y1 when interpreted within $\Lambda \mathrm{CDM}$ and its resolution after accounting for clumping. The right panel shows how M1 and M2 simultaneously alleviate the $H_{0}$ and $S_{8}$ tensions. The shaded vertical regions show the $68 \%$ and $95 \%$ C.L. bands of $H_{0}$ from SH0ES [3]. 
$\sim 70.5 \mathrm{~km} \mathrm{~s}^{-1} \mathrm{Mpc}^{-1}$ for M1. This is because the same shift in the sound horizon requires a smaller adjustment of $H_{0}$ to preserve the angular scale of the acoustic feature measured at low redshifts of $z \sim 0.5$ compared to that at $z \sim 1000$. We note that adding the new parameter $b$ results in only minor changes to the tightness of the posterior distributions of $H_{0}$ and other $\Lambda \mathrm{CDM}$ parameters (see the Supplemental Material [32]). This shows that clumping really solves the tensions, as opposed to simply allowing more parameter freedom to accommodate independent datasets.

We have so far assumed that the mildly nonlinear clumping is due to the existence of PMFs. Are there alternative sources of baryon clumping? An excess of small-scale power of inflationary adiabatic perturbations would be erased by Silk damping. The situation is different for small-scale isocurvature baryon fluctuations that survive Silk damping but are, nevertheless, constrained by big bang nucleosynthesis [41]. Taking the precise inferences of the primordial deuterium abundance from quasar absorption line systems at face value $[42,43]$, even mildly nonlinear isocurvature fluctuations should be ruled out. PMFs have the advantage that they survive Silk damping $[44,45]$ and actively source the generation of inhomogeneities only shortly before recombination and not during the big bang nucleosynthesis. The same may apply for cosmic string loops or accreting PBHs, however, it is questionable if they can produce the large volume filling baryon clumping as observed with PMFs.

Magnetic fields are ubiquitous in the universe, observed essentially in all astrophysical environments, including micro-Gauss $(\mu \mathrm{G})$ fields in galaxies and clusters of galaxies. Whereas magnetic fields coherent on galactic scales are often believed to result from the dynamo amplification of a pre-existing seed field [46], such as that produced in shocks during the collapse of the galaxy, the origin of cluster magnetic fields could possibly be explained by an interplay of galactic dynamos and outflows. More difficult is the explanation of $\sim \mu \mathrm{G}$ fields in protogalaxies too young to have gone through the number of revolutions necessary for the dynamo to work [47]. There is also indirect evidence from observations of $\gamma$ rays from $\mathrm{TeV}$ blazars for the existence of an essentially volume filling magnetic field in cosmic voids [48-50]. Since the field strength is likely weak, such fields may possibly be explained by the combined action of outflows from many galaxies. However, none of these astrophysical explanations are well understood, or have been explicitly shown to work. A magnetic field capable of generating baryon density fluctuations with $b \approx 0.5$ corresponds to a prerecombination PMF of $\sim 0.07 \mathrm{nG}$ comoving strength [25]. The PMF that survives to low redshifts may still be a factor of 6 less (for nonhelical fields), depending on the magnetogenesis scenario, resulting in $\sim 0.01 \mathrm{nG}$ prestructure formation fields (see discussion below). Such field strengths are just of the right order to explain the cluster fields, which require a precollapse magnetic field of $\sim 0.005 \mathrm{nG}$ irrespective of its coherence length [51-53]. Thus, a discovery of PMFs at recombination of this strength would have the stunning byproduct of explaining entirely the galactic and cluster magnetic fields, and the fields in the extragalactic medium.

PMFs could have been generated during cosmological first order phase transitions in the early Universe [54], during inflation [55,56], or at the end of inflation [57] (see Ref. [58] for a review). A detection of the PMF would offer an invaluable insight into the physics of the early Universe $[59,60]$. Inflationary models of magnetogenesis $[55,56]$, by nature, have to result in an approximately scale-invariant PMF spectrum not to be ruled out. PMFs generated via causal processes during phase transitions always develop a unique blue Batchelor spectra with most power on very small scales [61,62]. Once produced, their subsequent evolution is much more dramatic than that of the inflationary magnetic fields $[27,63,64]$. Because of the high conductivity in the early Universe, magnetic helicity is essentially conserved, whereas magnetic energy gets dissipated. A causally produced PMF of considerable strength will dissipate many orders of magnitude of its total initial energy density prior to recombination, and a factor of $\sim 20-40$ during or after recombination, depending on the helicity. In general, fields of even a small initial magnetic helicity will develop to be maximally helical during the course of the evolution and decay slower afterwards compared to their nonhelical counterparts. It is generally believed that the cosmological electroweak transition, in the best case, may only produce prerecombination fields of $\sim 0.1 \mathrm{nG}$ when some initial helicity is present [65]. Interestingly, nonzero helicity has been linked to the possible generation of the baryon asymmetry during the electroweak transition [66], although the predicted helicity, in units of the maximal one, can be at most $h_{m} \sim 10^{-24}$ [65]. However, a much larger helicity of $h_{m} \sim 10^{-3}$ is required to achieve $\sim 0.1 \mathrm{nG}$ fields before recombination (see Fig. 19 of Ref. [27]). In this context, new developments in chiral MHD linking helicity to left-right-handed particle asymmetries are worth noting $[67,68]$. On the other hand, the mere presence of PMFs during the electroweak transition has impact on the efficiency of baryogenesis itself $[69,70]$. Last, but not least, the conclusions regarding the requirements to produce $\sim 0.1 \mathrm{nG}$ fields may be changed by the possible discovery of magnetic inverse cascades in nonhelical magnetic fields $[71,72]$.

It is our belief that amending $\Lambda \mathrm{CDM}$ by clumping due to magnetic fields is a very modest and physically reasonable extension that shows promise to resolve the existing tensions. Ultimately, the existence of PMFs may have to be established by further smoking guns in future observations. For causally produced fields, an initial estimate shows that a future mission like the primordial inflation 
explorer (PIXIE) [73], targeting the spectral distortions of $\mathrm{CMB}$, is sensitive enough to detect the dissipation of magnetic fields at redshifts $z \sim 10^{4}$. A competing effect may be distortions in the spectrum due to Silk damping [74]. The metric fluctuations induced by causal PMFs do not make a detectable contribution to anisotropies in the $\mathrm{CMB}$, while a scale-invariant PMF can only make a detectable impact if the field is of $\sim 1 \mathrm{nG}$ strength [75-81] - at least an order of magnitude stronger than that required to produce a detectable clumping signature. The next generation $\mathrm{CMB}$ polarization experiments, such as probe of inflation and cosmic origins (PICO) [82] and CMB-S4 [83], can probe scale-invariant PMFs of $\sim 0.1 \mathrm{nG}$ strength through measurements of the Faraday rotation induced at the epoch of last scattering [84]. Additional constraints on large scale magnetic fields come from observations of cosmic rays $[85,86]$.

In this Letter, we have shown that mildly nonlinear, small-scale baryon inhomogeneities in the Universe existing before recombination, as expected to naturally emerge from $\sim 0.1 \mathrm{nG}$ prerecombination PMFs [24,25], show promise to resolve the Hubble tension. Such scenarios result in an inevitable reduction of the sound horizon at recombination, and thereby may bring local measurements of $H_{0}$ in agreement with the inferences from $\mathrm{CMB}$. Interestingly, if PMFs of such strength existed, the origin of galactic, cluster, and extragalactic magnetic fields would be explained.

We thank Antony Lewis, Simone Peirone, Vivian Poulin, Marco Raveri, Andrey Saveliev, Meir Shimon, Tanmay Vachaspati, Alex Zucca, and Gong-Bo Zhao for valuable assistance and discussions. We gratefully acknowledge using GetDist [87]. This research was enabled in part by support provided by SciNet and Compute Canada. L. P. is supported in part by the National Sciences and Engineering Research Council (NSERC) of Canada.

*karsten.jedamzik@umontpellier.fr

†levon@sfu.ca

[1] N. Aghanim et al. (Planck Collaboration), Astron. Astrophys. 641, A6 (2020).

[2] T. M. C. Abbott et al. (DES Collaboration), Mon. Not. R. Astron. Soc. 480, 3879 (2018).

[3] M. Reid, D. Pesce, and A. Riess, Astrophys. J. Lett. 886, L27 (2019).

[4] C. Potter, J. B. Jensen, J. Blakeslee, P. Milne, P. M. Garnavich, and P. Brown, in American Astronomical Society Meeting Abstracts \#232 (American Astronomical Society Meeting Abstracts, Washington DC, 2018), Vol. 232, p. 319.02

[5] W. L. Freedman et al., arXiv:1907.05922.

[6] W. Yuan, A. G. Riess, L. M. Macri, S. Casertano, and D. Scolnic, Astrophys. J. 886, 61 (2019).

[7] C. D. Huang et al., Astrophys. J. 889, 5 (2020).
[8] L. Verde, T. Treu, and A. G. Riess, Nat. Astron. 3, 891 (2019).

[9] M. J. Reid, J. A. Braatz, J. J. Condon, L. J. Greenhill, C. Henkel, and K. Y. Lo, Astrophys. J. 695, 287 (2009).

[10] D. Pesce et al., Astrophys. J. 891, L1 (2020).

[11] K. C. Wong et al., arXiv:1907.04869.

[12] T. M. C. Abbott et al. (DES Collaboration), Phys. Rev. D 98, 043526 (2018).

[13] L. Knox and M. Millea, Phys. Rev. D 101, 043533 (2020).

[14] G.-B. Zhao et al., Nat. Astron. 1, 627 (2017).

[15] Y. Wang, L. Pogosian, G.-B. Zhao, and A. Zucca, Astrophys. J. 869, L8 (2018).

[16] M. Raveri, Phys. Rev. D 101, 083524 (2020).

[17] E. Di Valentino, A. Melchiorri, O. Mena, and S. Vagnozzi, Phys. Rev. D 101, 063502 (2020).

[18] V. Poulin, T. L. Smith, T. Karwal, and M. Kamionkowski, Phys. Rev. Lett. 122, 221301 (2019).

[19] P. Agrawal, F.-Y. Cyr-Racine, D. Pinner, and L. Randall, arXiv:1904.01016.

[20] M.-X. Lin, G. Benevento, W. Hu, and M. Raveri, Phys. Rev. D 100, 063542 (2019).

[21] J. Sakstein and M. Trodden, Phys. Rev. Lett. 124, 161301 (2020).

[22] J. C. Hill, E. McDonough, M.W. Toomey, and S. Alexander, Phys. Rev. D 102, 043507 (2020).

[23] C. D. Kreisch, F.-Y. Cyr-Racine, and O. Dore, Phys. Rev. D 101, 123505 (2020).

[24] K. Jedamzik and T. Abel, J. Cosmol. Astropart. Phys. 10 (2013) 050.

[25] K. Jedamzik and A. Saveliev, Phys. Rev. Lett. 123, 021301 (2019).

[26] In this Letter, the field strengths are quoted at their comoving redshifted present day values.

[27] R. Banerjee and K. Jedamzik, Phys. Rev. D 70, 123003 (2004).

[28] A. Lewis, A. Challinor, and A. Lasenby, Astrophys. J. 538, 473 (2000).

[29] S. Seager, D. D. Sasselov, and D. Scott, Astrophys. J. 523, L1 (1999).

[30] S. Seager, D. D. Sasselov, and D. Scott, Astrophys. J. Suppl. 128, 407 (2000).

[31] W. Y. Wong, A. Moss, and D. Scott, Mon. Not. R. Astron. Soc. 386, 1023 (2008).

[32] See Supplemental Material at http://link.aps.org/ supplemental/10.1103/PhysRevLett.125.181302 for details of the employed three-zone models, comment on the relationship of this work to [25] and provide additional fits and cosmological parameters.

[33] A. Lewis and S. Bridle,Phys. Rev. D 66, 103511 (2002).

[34] N. Aghanim et al. (Planck Collaboration), Astron. Astrophys. 641, A5 (2020).

[35] F. Beutler, C. Blake, M. Colless, D. H. Jones, L. StaveleySmith, L. Campbell, Q. Parker, W. Saunders, and F. Watson, Mon. Not. R. Astron. Soc. 416, 3017 (2011).

[36] A. J. Ross, L. Samushia, C. Howlett, W. J. Percival, A. Burden, and M. Manera, Mon. Not. R. Astron. Soc. 449, 835 (2015).

[37] S. Alam et al. (BOSS Collaboration), Mon. Not. R. Astron. Soc. 470, 2617 (2017). 
[38] M. Blomqvist et al., Astron. Astrophys. 629, A86 (2019).

[39] D. Scolnic et al., Astrophys. J. 859, 101 (2018).

[40] We treat the BAO data with caution until the potential bias due to using a $\Lambda \mathrm{CDM}$ based template in BAO likelihoods is estimated for our class of models. During the presubmission stages of our paper a relevant study examining this issue for several "exotic" alternatives to $\Lambda$ CDM appeared [88], finding that the bias is negligible for the models they considered. Confirming this for baryon clumping is left for a future study.

[41] K. Jedamzik and G. M. Fuller, Astrophys. J. 452, 33 (1995).

[42] R. J. Cooke, M. Pettini, K. M. Nollett, and R. Jorgenson, Astrophys. J. 830, 148 (2016).

[43] S. Balashev, E. Zavarygin, A. Ivanchik, K. Telikova, and D. Varshalovich, Mon. Not. R. Astron. Soc. 458, 2188 (2016).

[44] K. Jedamzik, V. Katalinic, and A. V. Olinto, Phys. Rev. D 57, 3264 (1998).

[45] K. Subramanian and J. D. Barrow, Phys. Rev. D 58, 083502 (1998).

[46] K. Subramanian, Galaxies 7, 47 (2019).

[47] R. M. Athreya, V. K. Kapahi, P. J. McCarthy, and W. van Breugel, Astron. Astrophys. 329, 809 (1998).

[48] A. Neronov and I. Vovk, Science 328, 73 (2010).

[49] H. Tashiro, W. Chen, F. Ferrer, and T. Vachaspati, Mon. Not. R. Astron. Soc. 445, L41 (2014).

[50] W. Chen, J. H. Buckley, and F. Ferrer, Phys. Rev. Lett. 115, 211103 (2015).

[51] R. Banerjee and K. Jedamzik, Phys. Rev. Lett. 91, 251301 (2003); 93, 179901(E) (2004).

[52] K. Dolag, M. Bartelmann, and H. Lesch, Astron. Astrophys. 348, 351 (1999).

[53] K. Dolag, M. Bartelmann, and H. Lesch, Astron. Astrophys. 387, 383 (2002).

[54] T. Vachaspati, Phys. Lett. B 265, 258 (1991).

[55] M. S. Turner and L. M. Widrow, Phys. Rev. D 37, 2743 (1988).

[56] B. Ratra, Astrophys. J. 391, L1 (1992).

[57] A. Diaz-Gil, J. Garcia-Bellido, M. Garcia Perez, and A. Gonzalez-Arroyo, Phys. Rev. Lett. 100, 241301 (2008).

[58] R. Durrer and A. Neronov, Astron. Astrophys. Rev. 21, 62 (2013).

[59] K. Subramanian, Rep. Prog. Phys. 79, 076901 (2016).

[60] T. Vachaspati, Phys. Rev. D 95, 063505 (2017).

[61] R. Durrer and C. Caprini, J. Cosmol. Astropart. Phys. 11 (2003) 010.
[62] A. Saveliev, K. Jedamzik, and G. Sigl, Phys. Rev. D 86, 103010 (2012).

[63] L. Campanelli, Phys. Rev. Lett. 98, 251302 (2007).

[64] L. Campanelli, Eur. Phys. J. C 74, 2690 (2014).

[65] J. M. Wagstaff and R. Banerjee, J. Cosmol. Astropart. Phys. 01 (2016) 002

[66] T. Vachaspati, Phys. Rev. Lett. 87, 251302 (2001).

[67] A. Boyarsky, J. Frohlich, and O. Ruchayskiy, Phys. Rev. Lett. 108, 031301 (2012).

[68] J. Schober, A. Brandenburg, and I. Rogachevskii, Geophys. Astrophys. Fluid Dyn. 114, 106 (2020).

[69] D. Comelli, D. Grasso, M. Pietroni, and A. Riotto, Phys. Lett. B 458, 304 (1999).

[70] A. De Simone, G. Nardini, M. Quiros, and A. Riotto, J. Cosmol. Astropart. Phys. 10 (2011) 030.

[71] A. Brandenburg, T. Kahniashvili, and A. G. Tevzadze, Phys. Rev. Lett. 114, 075001 (2015).

[72] J. Zrake, Astrophys. J. 794, L26 (2014).

[73] A. Kogut and et al, J. Cosmol. Astropart. Phys. 11 (2011) 025.

[74] J. Chluba et al., arXiv:1909.01593.

[75] T. R. Seshadri and K. Subramanian, Phys. Rev. Lett. 87, 101301 (2001).

[76] A. Mack, T. Kahniashvili, and A. Kosowsky, Phys. Rev. D 65, 123004 (2002).

[77] A. Lewis, Phys. Rev. D 70, 043011 (2004).

[78] F. Finelli, F. Paci, and D. Paoletti, Phys. Rev. D 78, 023510 (2008).

[79] J. R. Shaw and A. Lewis, Phys. Rev. D 81, 043517 (2010).

[80] P. A. R. Ade et al. (Planck Collaboration), Astron. Astrophys. 594, A19 (2016).

[81] A. Zucca, Y. Li, and L. Pogosian, Phys. Rev. D 95, 063506 (2017).

[82] S. Hanany et al. (NASA PICO Collaboration), arXiv: 1902.10541.

[83] K. Abazajian et al., arXiv:1907.04473.

[84] L. Pogosian, M. Shimon, M. Mewes, and B. Keating, Phys. Rev. D 100, 023507 (2019).

[85] S. Archambault et al. (VERITAS Collaboration), Astrophys. J. 835, 288 (2017).

[86] P. Tiede, A. E. Broderick, M. Shalaby, C. Pfrommer, E. Puchwein, P. Chang, and A. Lamberts, arXiv:1702.02586.

[87] A. Lewis, arXiv:1910.13970.

[88] J. L. Bernal, T. L. Smith, K. K. Boddy, and M. Kamionkowski, arXiv:2004.07263. 